Submitted to the $29^{\text {th }}$ International Symposium on Combustion

\title{
SPATIALLY-RESOLVED SMALL-ANGLE X-RAY SCATTERING STUDIES OF SOOT INCEPTION AND GROWTH
}

\author{
J. P. HESSLER, S. SEIFERT, AND R.E. WINANS \\ Chemistry Division \\ Argonne National Laboratory \\ 9700 South Cass Avenue \\ Argonne, Illinois 60439-4831, U.S.A. \\ Corresponding Author: $\quad$ Dr. Jan P. Hessler \\ Chemistry Division \\ Argonne National Laboratory \\ 9700 South Cass Avenue \\ Argonne, Illinois 60439-4831, U.S.A. \\ Facsimile: 1-630-252-4470 Voice: 1-630-252-3717 \\ E-mail: $\quad$ hessler@anl.gov \\ Presentation Mode: Oral Presentation \\ Colloquium: $\quad$ Pollutant Formation and Control (NOx, SOx, PAH, Soot, Air Toxics) \\ Keyword: $\quad$ SOOT \\ Total Word Count: $\quad 5031$ \\ Text: $\quad 196$ lines@15 words/line 2940 \\ Equations: 5 equations @ 21 words/equation 105 \\ Fig. 1: one column wide @ 2.50" high 200 \\ Fig. 2: one column wide @ 3.00" high 240 \\ Fig. 3: one column wide@ 2.00" high 160 \\ Fig. 4: one column wide @ 2.00" high 160 \\ Fig. 5: one column wide @ 3.50" high 280 \\ Fig. 6: two column wide @ 4.25" high 680 \\ References: 38 lines@7 words/line 266
}

The submitted manuscript has been created by the University of Chicago as Operator of Argonne National Laboratory ("Argonne") under Contract No. W-31-109-ENG-38 with the U.S. Department of Energy. The U.S. Government retains for itself, and others acting on its behalf, a paid-up, nonexclusive, irrevocable worldwide license in said article to reproduce, prepare derivative works, distribute copies to the public, and perform publicly and display publicly, by or on behalf of the Government.

This work was performed under the auspices of the U. S. Department of Energy, Office of Basic Energy Sciences, Division of Chemical Sciences, Geosciences, and Biosciences and use of the Advanced Photon Source was supported by BES-DOE, all under contract number W-31-109ENG-38. 


\title{
SPATIALLY-RESOLVED SMALL-ANGLE X-RAY SCATTERING STUDIES OF SOOT INCEPTION AND GROWTH
}

\author{
J. P. HESSLER, S. SEIFERT, AND R.E. WINANS \\ Chemistry Division \\ Argonne National Laboratory \\ 9700 South Cass Avenue \\ Argonne, Illinois 60439-4831, U.S.A.
}

\begin{abstract}
The high spectral brilliance of x-rays produced at the Basic Energy Sciences Synchrotron Radiation Center of Argonne's Advanced Photon Source allows us to perform small-angle x-ray scattering (SAXS) measurements of the distributions of soot particles in flames. SAXS provides an in situ probe of the size and distribution of particles in the region between 1 and $100 \mathrm{~nm}$. Detailed measurements on a propylene/air diffusion flame allow us to extract a spatiallydependent background, which occurs in gas-phase combustion systems, and to perform Abel inversions, which provide the radial dependence of the scattering intensity. A bimodal distribution of soot particles is needed to describe our results. The radial behavior of the two modes of this distribution implies that the chemistry and fluid dynamics are strongly coupled in this simple diffusion flame. The larger particles of this distribution correspond to the previously observed primary particles, which have a relatively complex radial dependence. Midway between the fuel source and the widest part of the flame the primary particles have a mean radius of $6 \mathrm{~nm}$ or less and their concentration is symmetrically distributed about the flame front. At the widest part of the flame, two distinct distributions of primary particles are observed. Near the center of the flame the particles have a mean radius of $10 \mathrm{~nm}$ and a polydispersity of 0.3 and beyond a transition region they have a mean radius of $21 \mathrm{~nm}$ and a polydispersity of 0.2 . The smaller particles, which require additional experiments before they can be identified, correspond either to soot nuclei, PAH species such as naphthalene, and/or disordered carbons with graphitic basal planes.
\end{abstract}

\section{Introduction}

A comprehensive model that is capable of predicting the inception and growth of soot over a wide range of chemical and physical conditions is just beginning to emerge and has been documented in various workshops[1] and symposia[2]. Here we report the first spatiallyresolved small-angle $\mathrm{x}$-ray scattering (SAXS) studies of soot formed during a relatively simple combustion process. Generally, in situ measurements have been performed with light in the ultraviolet, optical, or infrared spectral regions and include absorption[3], induced[4] and 
natural[5] incandescence, and wide-angle elastic scattering[6]. Scattering measurements provide information on the morphology of particles that are larger than $15 \mathrm{~nm}$ while absorption and incandescence measurements have been used to detect particles with mean radii between 1.8 and $10 \mathrm{~nm}$. Combined scattering and extinction measurements in the ultraviolet-visible region have detected particles in the 2-3 and 8-10 nm ranges.[7] Thermophoretic sampling followed by transmission electron microscopy[8] and differential mobility analysis[9] have been used to observe soot particles with mean diameters between 3 and $18 \mathrm{~nm}$, but these are ex situ techniques. SAXS is ideally suited to provide in situ measurements in the important region between 1 and $100 \mathrm{~nm} .[10]$ In 1986, W. A. England presented the first in situ SAXS studies of soot morphology.[11] He used a second-generation x-ray source and his exposure times had to be quite long. Third-generation x-ray sources, such as the Advanced Photon Source at Argonne National Laboratory, contain insertion devices, in our case an undulator, that increase the spectral brilliance of the $\mathrm{x}$-rays by as much as a factor of 5000 compared to a bending magnet.[12] Because of this dramatic increase in brilliance, only a few seconds are needed to obtain a scattering profile from soot particles formed in a small flame. Recently, we reported our first measurements of SAXS from soot formed in both an acetylene diffusion flame and a toluene flame in a burner that supports a CH4/H2/air diffusion flame.[13] We observed numerous "dips" in the scattering profiles that could not be explained with standard calculations or models. Here, we present new and more detailed measurements on a propylene diffusion flame that allow us to identify and extract a spatially-dependent background, which we attribute to a temperature/density gradient that is produced by the flame front, and the axial and radial dependence of the scattering profiles. We can describe our results in terms of a bimodal distribution of soot particles. The larger particles of this distribution correspond to polydispersed 
primary particles, with properties that depend upon both the radial and axial position within the flame, and that ultimately lead to a distribution with a mean radius of gyration, $<\mathrm{R}_{\mathrm{g}}>$, of $21 \mathrm{~nm}$ and a polydispersity, $\sigma_{\mathrm{S}} /\left\langle\mathrm{R}_{\mathrm{g}}\right\rangle$, of 0.2 , where $\sigma_{\mathrm{S}}$ is the width of the Schultz distribution[14]. The smaller particles, which require additional experiments before they can be identified, may be the soot nuclei observed by di Stasio[15], a polycyclic aromatic hydrocarbon such as naphthalene, which we have observed[16], and/or disordered carbons with graphitic basal planes observed by Chen and Dobbins[17].

\section{Experimental Methods}

SAXS is over sixty years old and dates from the classical works of Guinier, which were published in 1938 and summarized in the book by Guinier and Fournet.[18] Subsequent texts cover the more modern aspects. $[19,20,21]$ All scattering results may be described in terms of the differential scattering cross section, $\mathrm{d} \sigma / \mathrm{d} \Omega$, which is given by

$$
\frac{d \sigma}{d \Omega}=\frac{S(\theta)}{I_{0}}=I(q)
$$

where $S(\theta)$ is the average energy scattered per unit time per unit solid angle at the angle $\theta$ with respect to the incident beam and $I_{0}$ is the intensity (energy time $\mathrm{e}^{-1}$ length ${ }^{-2}$ ) of the incident beam of x-rays. The differential scattering cross section, which is generally referred to as the scattering intensity by people who work with x-rays, is denoted by I(q) where q is the transferred momentum. The dimension of $\mathrm{I}(\mathrm{q})$ is length ${ }^{2}$, even though it is referred to as an intensity. The transferred momentum is given by $\mathrm{q}=(4 \pi / \lambda) \sin (\theta / 2)$ where $\lambda$ is the wavelength of the incident $\mathrm{x}$-rays. The scattering intensity is formally given by

$$
I(q)=V \int_{V}\left\langle\rho\left(r_{0}\right) \rho\left(r_{0}+r\right)\right\rangle \exp (-i q \bullet r) d r
$$


where $<\rho\left(r_{0}\right) \rho\left(r_{0}+r\right)>$ is the autocorrelation function and expresses the spatial dependence of the self-correlation of the electron density, $\rho(\mathrm{r})$, averaged over the total irradiated volume $\mathrm{V}$. Structural information on the particles is obtained by measuring the scattering intensity over a sufficiently large range of transferred momentum. Practically, this involves performing experiments with x-rays at different energies (wavelengths) between 6 and $18 \mathrm{keV}(0.21$ and $0.069 \mathrm{~nm})$ and with different distances between the flame and detector. Different regions of transferred momentum provide different information about the system. In the Guinier region, $\mathrm{q}<\mathrm{R}_{\mathrm{g}}><<1$, we can extract only the mean radius of gyration of the particles. Above $\mathrm{q}<\mathrm{R}_{\mathrm{g}}>\sim 1$, the Porod region, the scattering intensity scales as $1 / \mathrm{q}^{4}$ and, if the system is not too polydispersed, interference fringes may be observed that will directly provide $<\mathrm{Rg}_{\mathrm{g}}>$ and the polydispersity, $\sigma /<\mathrm{R}_{\mathrm{g}}>$, where $\sigma$ is the width of the distribution. Scattering from soot particles has multiple length scales and, therefore, we anticipate that each of these regions will be present for soot nuclei, primary particles, and aggregates. Unfortunately, these regions may overlap and, at the present time, analytic expressions that join the different regions simply do not exist.

Our SAXS apparatus has been described previously.[22] Briefly, it consists of a high brilliance undulator, a double-crystal monochromator, and the components needed to transport, focus, define, and monitor the incident beam of $\mathrm{x}$-rays. Typically, $10^{12} \mathrm{x}$-ray photons per second in a beam about $100 \mu \mathrm{m}$ in diameter are incident on the sample, which is located in the experimental hutch 12 ID-C of the Basic Energy Sciences Synchrotron Radiation Center. The fuel, in this case propylene, is injected through a $500 \mu \mathrm{m}$ i.d. tube into the center of a $25.4 \mathrm{~mm} \mathrm{x}$ $25.4 \mathrm{~mm}$ flat-flame burner, which contains an array of $1 \mathrm{~mm}$ diameter tubes, that is used to introduce air. This burner is surrounded by a $6.3 \mathrm{~mm}$ wide co-flow region, which is also constructed with $1 \mathrm{~mm}$ diameter tubes, that is used to isolate the system from molecules in the 
laboratory by providing a flowing stream of nitrogen molecules.[23] A stable cylindrical flame that showed incandescence up to a height of approximately $100 \mathrm{~mm}$ was obtained with flow rates of 0.090, 4.0, and 14.6 SLPM for propylene, air, and nitrogen, respectively. One-hundred-thirtysix SAXS profiles were obtained at each of thirty-three different heights (4488 profiles) above the tip of the burner by using two linear translation stages to move the flame with respect to the beam of $x$-rays.

The luminous flame boundary of a diffusion flame, which is generally defined by fluorescence from the $\mathrm{CH}$ radical, may be used to define two regions of the flame. The inner region is fuel rich and where soot formation dominates, while in the outer region oxidation dominates. Therefore, soot volume fractions, mean particle diameters, and soot concentrations all depend critically upon both the radial and axial position within the flame.[24] Our measured scattering profile is an integral of the local scattering intensity, which we refer to as the integrated scattering intensity, and may be written

$$
I(q, X)=2 \int_{X}^{\infty} \frac{r \bullet i(q, r)}{\left(r^{2}-X^{2}\right)^{1 / 2}} d r
$$

where $\mathrm{I}(\mathrm{q}, \mathrm{X})$ is the integrated scattering intensity at the transferred momentum $\mathrm{q}$ in the laboratory coordinate system and $\mathrm{i}(\mathrm{q}, \mathrm{r})$ is the local scattering intensity at the same transferred momentum in the cylindrical coordinate system centered on the flame. $\mathrm{X}$ is the distance from the center of the flame in the laboratory system and $r$ the distance in the cylindrical system. The local scattering intensity is obtained by inverting the measured scattering profiles. Because of the cylindrical symmetry of our flames, this inversion is performed by the Abel transformation

$$
i(q, r)=-\frac{1}{\pi} \int_{r}^{\infty} \frac{[d I(q, X) / d X]}{\left(X^{2}-r^{2}\right)^{1 / 2}} d X .
$$

This transform has been discussed by many authors; we use Dasch's approach[25]. 


\section{Results and Discussion}

In this work we report two sets of measurements on a propylene diffusion flame in air. The first covers the range $0.2<\mathrm{q}\left(\mathrm{nm}^{-1}\right)<6$ and is referred to as a high-q experiment, while the second covers the range $0.06<\mathrm{q}\left(\mathrm{nm}^{-1}\right)<1.9$ and is referred to as a medium-q experiment. In fig. 1 we show the measured scattering intensity for a medium-q experiment as a function of position, $\mathrm{X}$, with respect to the center of the flame. A spatially-dependent background is observed at distances greater than $3 \mathrm{~mm}$ from the center of the flame. Typically, the standard deviation of a least-squares fit of this background to a parabolic expression, $\mathrm{A}(\mathrm{q})+\mathrm{B}(\mathrm{q}) \mathrm{X}^{2}$, is less than $2 \%$ of the spatially independent part of the background, i.e. $\sigma(q) / A(q)<0.02$ for all values q. The sources of background scattering are the apparatus and molecules such as nitrogen, oxygen, and the fuel. The temperature gradient that is present near the flame must be accompanied by a density gradient and this gradient implies that the background scattering must be spatially dependent. We approximate this dependence by a parabolic expression and subtract it before we perform the Abel inversion.

Figure 2 shows the local scattering intensity, calculated by equation (4), for a high-q experiment as a function of transferred momentum for several different radial positions. These data were taken near the widest and brightest part of the flame, approximately $20 \mathrm{~mm}$ above the tip of the fuel inlet. The major conclusion we drawn from this figure, and many others like it, is that we are observing scattering from at least two different species of soot. For values of $\mathrm{q}$ greater than $1.0 \mathrm{~nm}^{-1}$ the relatively constant signal may be described by Guinier's equation[13], $\operatorname{Gexp}\left\{-\mathrm{q}^{2} \mathrm{R}_{\mathrm{g}}^{2} / 3\right\}$, with $\mathrm{R}_{\mathrm{g}} \sim 0.2 \mathrm{~nm}$. Another crucial observation is that the prefactor, $\mathrm{G}$, which is directly proportional to the concentration of the species responsible for scattering in this region, is independent of the scattering intensity observed for $\mathrm{q}<1 \mathrm{~nm}^{-1}$. This independence is clearly 
illustrated by the solid triangles $(r=0.1 \mathrm{~mm})$, open circles $(r=0.5 \mathrm{~mm})$, and solid circles $(r=0.8$ $\mathrm{mm})$. For $\mathrm{q}<0.5 \mathrm{~nm}^{-1}$ the intensities of these three curves are nearly identical yet for $\mathrm{q}>2 \mathrm{~nm}^{-1}$ the triangles are at least a factor of three larger than the open circles and the solid circles are essentially zero. The facts that these two distributions are independent of one another and that scattering from the smaller species goes to zero at certain positions indicate that both the chemical reactions responsible for soot formation and the fluid dynamics that control the relative motion of species in the flame must be considered when simulating soot formation in a simple diffusion flame.

Before we discuss the nature of the species responsible for scattering at the highest $\mathrm{q}$ values, we consider results at lower q, where scattering from the primary particles is observed. Just below $\mathrm{q} \sim 0.6 \mathrm{~nm}^{-1}$ scattering from the primary particles is proportional to $1 / \mathrm{q}^{4}$. Scattering in this region is most easily discussed by generating a plot of $\log \left\{\mathrm{q}^{4} \mathrm{i}(\mathrm{q}, \mathrm{r})\right\}$ vs. $\log \{\mathrm{q}\}$, which amplifies subtle changes in the scattering intensity. Figure 3 shows a series of such plots for radial positions between 0.4 and $1.2 \mathrm{~mm}$ at an axial position midway between the tip of the fuel inlet and the widest part of the flame. The strongest profile $(\mathrm{r}=1.2 \mathrm{~mm})$ shows a weak dip near $0.5 \mathrm{~nm}^{-1}$. The location of this dip indicates that the particles at $1.2 \mathrm{~mm}$ have a mean radius of gyration of $6 \mathrm{~nm}$ or less. The relative concentrations of the primary particles and the smaller species may be obtained by calculating the Porod invariant,

$$
Q_{i n v}(r)=\int q^{2}\left[i_{p p}(q, r)+G \exp \left(-q^{2} R_{s}^{2} / 3\right)\right] d q
$$

where $\mathrm{i}_{\mathrm{pp}}(\mathrm{q}, \mathrm{r})$ is the scattering intensity from the primary particles and the second term represents scattering from the smaller species. These invariants for different radial positions at this height in the flame are shown in fig. 4. 
Just above the widest and brightest part of the flame the scattering profiles from the primary particles become more complex. This complexity is demonstrated in fig. 5 where we show plots of $\log \left\{\mathrm{q}^{4} \mathrm{i}(\mathrm{q}, \mathrm{r})\right\}$ vs. $\log \{\mathrm{q}\}$ for radii between 0.9 and $1.3 \mathrm{~mm}$. For $\mathrm{r}<0.9 \mathrm{~mm}$ all of the scattering profiles show a distinct minimum at $\mathrm{q} \sim 0.35 \mathrm{~nm}^{-1}$, which indicates that $\left\langle\mathrm{R}_{\mathrm{g}}>\sim 10\right.$ $\mathrm{nm}$; the depth of the modulation gives a polydispersity, $\sigma_{\mathrm{S}} /<\mathrm{Rg}>$, of 0.3 , where $\sigma_{\mathrm{S}}$ is the width of the Schultz distribution[14]. Between 0.9 and $1.3 \mathrm{~mm}$ from the flame center the scattering profiles systematically change to produce three dips. The first occurs at $\mathrm{q} \sim 0.2 \mathrm{~nm}^{-1}$, which implies that $\left\langle\mathrm{R}_{\mathrm{g}}>\sim 21 \mathrm{~nm}\right.$. The depth of this modulation suggests that these particles are not as dispersed; the polydispersity is 0.2 . It is instructive to compare our in situ results with the ex situ SAXS measured by Sorensen et al.[26] Their data, which have been scaled by a constant factor to fit on our graph, are shown as the solid dots in fig. 5. Their first minimum, at $\mathrm{q}=0.25 \mathrm{~nm}^{-1}$, indicates $<\mathrm{R}_{\mathrm{g}}>=18 \mathrm{~nm}$, and, for a Gaussian distribution, a polydispersity of 0.17 . Their scattering profiles in this region did not depend upon the source of the soot nor its treatment. Therefore, we conclude that the larger primary particles in our experiment represent mature primary particles. In fig. 6 we display the Porod invariant at different radial positions for the profiles shown in fig. 5. There are three, possibly four, distinct regions in this figure. From 0 to $0.9 \mathrm{~mm}$ the relative concentration of the smaller particles is large and the primary particles are relatively small and dispersed. Between 0.9 and $1.3 \mathrm{~mm}$ there is a transition region; the relative concentration of the smaller species appears to peak and the size of the primary particle increases by a factor of two while they become less dispersed. From 1.3 to $2.5 \mathrm{~mm}$ the relative concentration of primary particles dominates the scattering, the concentration of the smallest species is approximately constant, and the shape of the scattering profiles indicate that there is little change in the size and distribution of the primary particles. Above $2.5 \mathrm{~mm}$ we see what 
may be a fourth region where the smallest particles begin to dominate the scattering as we move away from the flame center.

Now that we have established that our results for primary soot particles agree with those of other researchers we consider the implications of our observations for $\mathrm{q}>1.0 \mathrm{~nm}^{-1}$. We have tested our apparatus by performing SAXS on $\mathrm{C}_{60}$, which has a radius of gyration of $0.363 \mathrm{~nm}$, and our results agree with neutron scattering measurements.[27] We can identify three different sources for scattering at $\mathrm{q}>1 \mathrm{~nm}^{-1}$ : the soot nuclei observed by di Stasio[15], a polycyclic hydrocarbon such as naphthalene[16], or disordered carbon with graphitic basal planes[17]. Chen and Dobbins have observed x-ray diffraction peaks in ex situ soot that are consistent with disordered carbons with graphitic basal planes. The positions of the peaks provide information about the spacing between the basal planes and their widths indicate the number of correlated planes. The only way to identify the source of this scattering is to measure the scattering intensity for $1<\mathrm{q}\left(\mathrm{nm}^{-1}\right)<40$. This will provide as complete a description of these scatters as is possible.

\section{Conclusions}

The results we have reported clearly demonstrate that small-angle x-rays scattering can provide detailed in situ information on the spatial dependence of the distribution of soot particles formed during combustion. This detailed information has allowed us to identify a bimodal distribution in the relative concentrations of the soot species and to map the spatial dependence of the soot species. Scattering from primary soot particles indicates that they have a relatively stable mean radius near $10 \mathrm{~nm}$ with a polydispersity of 0.3 and then undergo a transition to particles with a mean radius of $21 \mathrm{~nm}$ and a polydispersity of 0.2 . In addition, we observe a significant amount of scattering for transferred momentum greater than $1.0 \mathrm{~nm}^{-1}$. Preliminary 
calculations of the radius of gyration of molecular soot precursors confirm that we are indeed observing the initial stages of soot formation. We are now in a position to design experiments that will overlap previous measurements with optical techniques and address some of the fundamental issues of soot inception and growth.

\section{Acknowledgements}

This work would not have be possible without the help of the staff of the Basic Energy Sciences Synchrotron Radiation Center at Argonne's Advanced Photon source, in particular, Mark A. Beno, Mark S. Engbretson, Guy Jennings, Charles A. Kurtz, and Jennifer A. Linton. Helpful conversations with Albert F. Wagner and Stephen T. Pratt are gratefully acknowledged. We also thank Chris M. Sorensen and Thomas P. Rieker for sharing with us their previously published data. This work was performed under the auspices of the U. S. Department of Energy, Office of Basic Energy Sciences, Division of Chemical Sciences, Geosciences, and Biosciences and use of the Advanced Photon Source was supported by BES-DOE, all under contract number W-31-109ENG-38. 


\section{REFERENCES}

1. H. Bockhorn, Ed., Soot formation in Combustion, Mechanisms and Models, Springer-Verlag, Berlin, 1994 and earlier workshops referenced therein.

2. K. H. Homann and M. J. Wornat, co-chairs of the colloquium on soot formation and destruction, Proc. Comb. Inst. 28:2507-2658 (2000) and previous symposia.

3. A. M. Weiner and S. J. Harris, Combust. Flame 77:261 (1989) and references therein.

4. D. Woiki, A. Giesen, and P. Roth, Proc. Comb. Inst. 28:2531 (2000).

5. T. E. Parker, J. R. Morency, R. R. Foutter, and W. T. Rawlins, Combust. Flame 107:271 (1996).

6. S. di Stasio, P. Massoli, and M. Lazzaro, J. Aersol. Sci. 27:897 (1996).

7. B. M. Vaglieco, S. S. Merola, A. D'Anna, and A. D'Alessio, J. Quant. Spectros. Radia. Transfer 73:443 (2002).

8. R. A. Dobbins and C. M. Megaridis, Langumir 3:254 (1987).

9. H. Hepp and K. Siegmann, Combust. Flame 115:275 (1998).

10. C. E. Williams, R. P. May, and A. Guinier, X-ray Characterization of Materials (E. Lifshin, Ed.), Wiley-VCH, Weinheim, 1999, pp. 211-254.

11. W. A. England, Combust. Sci. Technol. 46:83 (1986).

12. U. W. Arndt, International Tables for Crystallography, Volume C (A.J.C. Wilson, Ed.), Kluwer Academic Pub., Dordrecht, 1992, pp. 172-175.

13. J. P. Hessler, S. Seifert, R. E. Winans, and T. H. Fletcher, Faraday Discuss. 119:395 (2001).

14. E. Y. Sheu, Phys. Rev. A 45:2428 (1992).

15. S. di Stasio, Carbon 39:109 (2001). 
16. R. E. Winans, S. Seifert, J. P. Hessler, to be presented at the $12^{\text {th }}$ International Conference on Small-Angle Scattering to be held in Venezia, Italy, August 25-29, 2002.

17. H. X. Chen, R. A. Dobbins, Comb. Sci. Technol. 159:109 (2000).

18. A. Guinier and G. Fournet, Small-Angle Scattering of X-rays, (translated into English by C. B. Walker), Wiley, New York and Chapman \& Hall, London, 1955.

19. Small-Angle X-ray Scattering, ed. O. Glatter and O. Kratke, Academic Press, London, 1982.

20. L. A. Feign and D.I. Svergun, Structure Analysis by Small-Angle X-ray and Neutron Scattering, Plenum Press, New York, 1987.

21. Modern Aspects of Small-Angle Scattering, ed. H. Brumberger, Kluwer, Dortrecht, 1995.

22. S. Seifert, R. E. Winans, D. M. Tiede, and P. Thiyagarajan, J. Appl. Crystallogr. 33:782 (2000).

23. J. Ma, T. H. Fletcher, and B. Webb, Energy Fuels 9:802 (1995).

24. R. J. Santoro, H. G. Semerjian, and R. A. Dobbins, Combust. Flame 51:203 (1983).

25. C. J. Dasch, Appl. Opt. 21:1146 (1992).

26. C. M. Sorenson, C. Oh, P. W. Schmidt, and T. P. Rieker, Phys. Rev. E 58:4666 (1998).

27. Y. B. Melnichenko, G. D. Wignall, R. N. Compton, and G. Bakale, J. Chem.. Phys. 111:4724 (1999). 


\section{Figure Captions:}

Fig. 1. Measured scattering intensity at a fixed value of the transferred momentum, $0.53 \mathrm{~nm}^{-1}$, as a function of laboratory position, $\mathrm{X}$, at a height of $20 \mathrm{~mm}$ above the flame tip during a medium-q experiment. The solid line is the parabola used to approximate the spatial dependence of the background scattering.

Fig. 2. Local scattering intensity, calculated by equation (4), as a function of transferred momentum at a height of $20 \mathrm{~mm}$ above the burner and at three different distances from the center of the flame: solid triangles $0.1 \mathrm{~mm}$, open circles $0.5 \mathrm{~mm}$, and solid circles $0.8 \mathrm{~mm}$.

Fig. 3. Plot of $\log \{\mathrm{q} 4 \mathrm{i}(\mathrm{q}, \mathrm{r})\}$ vs. $\log \{\mathrm{q}\}$ where $\mathrm{i}(\mathrm{q}, \mathrm{r})$ is the local scattering intensity at a height of $10 \mathrm{~mm}$ from the base of the flame and at radial position from 0.4 to $1.2 \mathrm{~mm}$ in steps of $0.1 \mathrm{~mm}$.

Fig 4. Radial dependence of the Porod invariant, equation (5), of the small species (solid circles) and primary soot particles (solid triangles) for the profiles shown in figure 3 . See the text for details.

Fig. 5. Plot of $\log \{\mathrm{q} 4 \mathrm{i}(\mathrm{q}, \mathrm{r})\}$ vs. $\log \{\mathrm{q}\}$ where $\mathrm{i}(\mathrm{q}, \mathrm{r})$ is the local scattering intensity at a height of $20 \mathrm{~mm}$ from the base of the flame and at radial positions $0.9,1.0,1.1,1.2$, and $1.3 \mathrm{~mm}$. See the text for details.

Fig 6. Radial dependence of the Porod invariant, equation (5), of the small species (solid circles) and primary soot particles (solid triangles) for the profiles shown in figure 5 . See the text for details. 
Figures

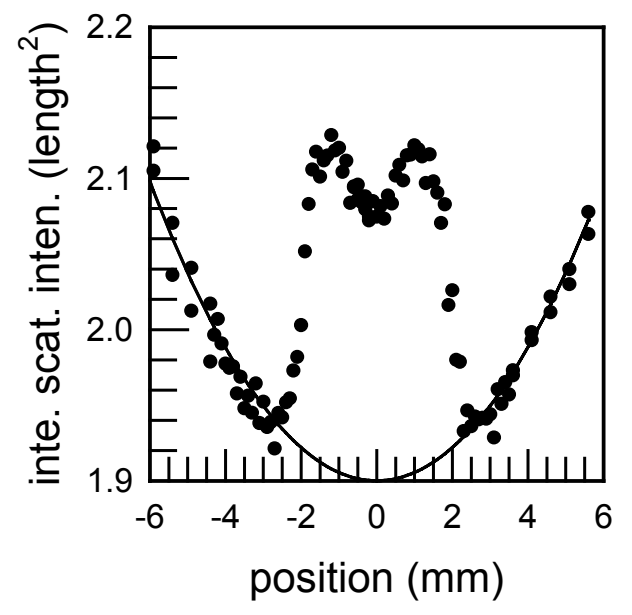

Figure 1.

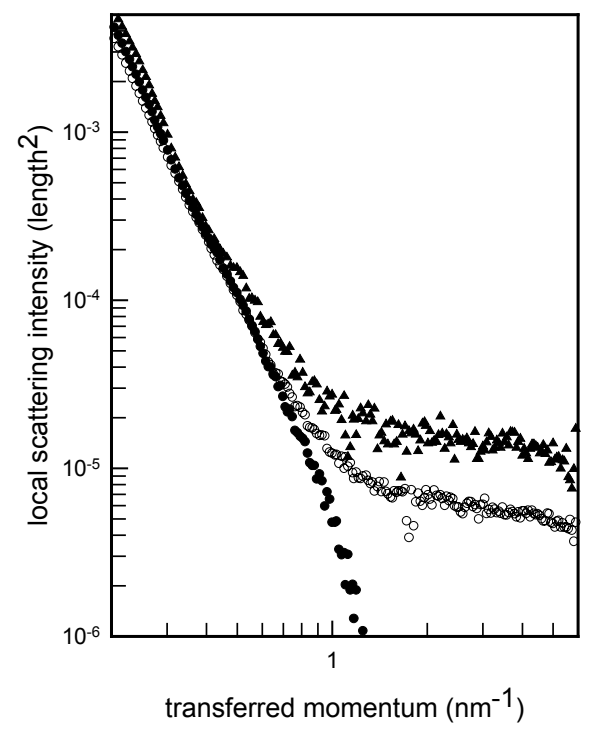

Figure 2. 


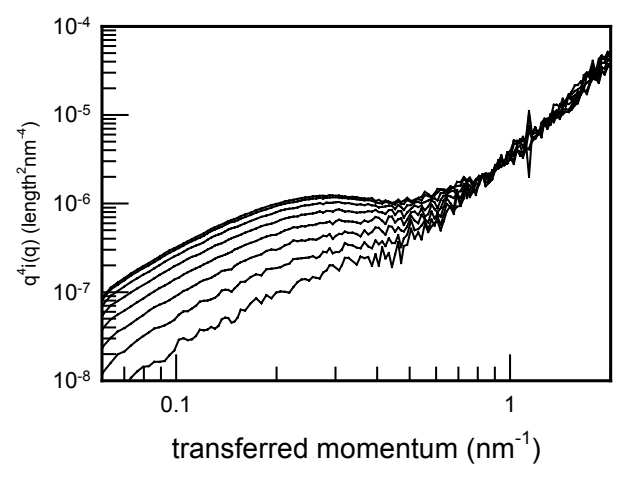

Figure 3.

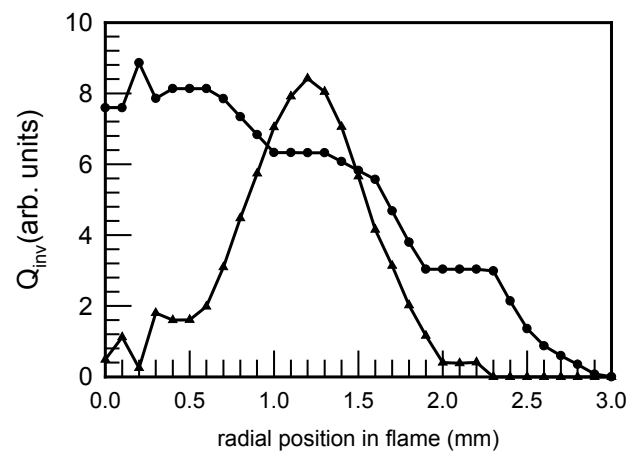

Figure 4. 


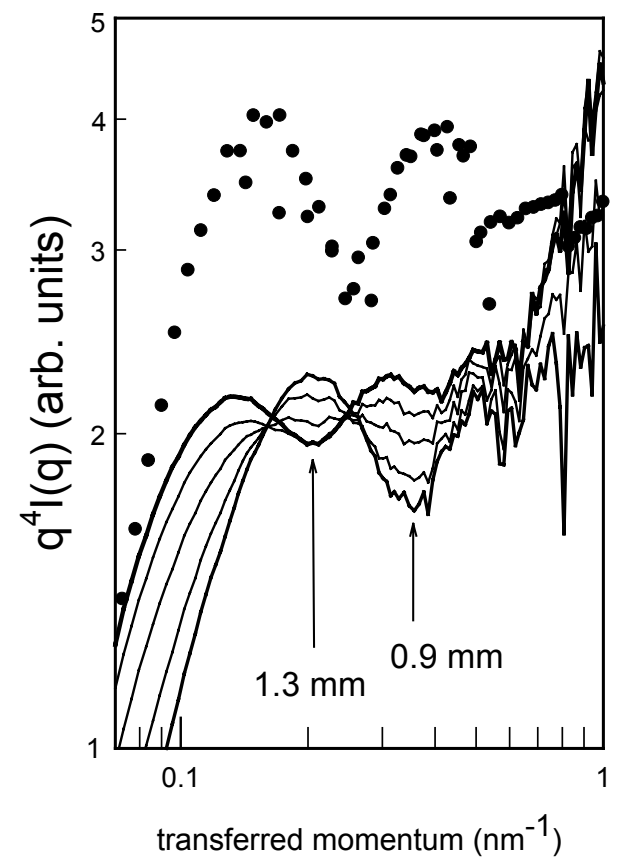

Figure 5. 


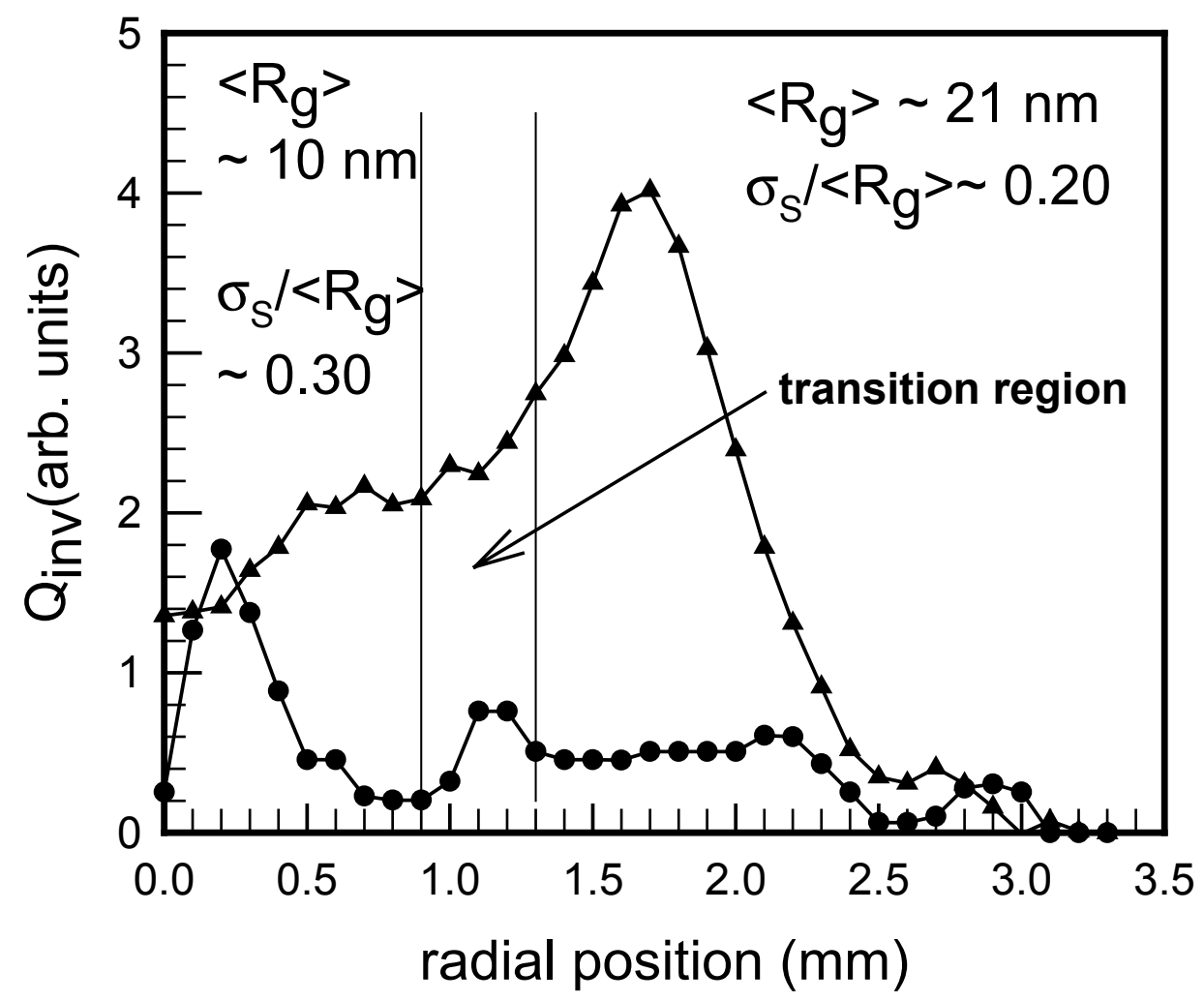

Figure 6. 